\title{
Efecto de la harina de coco desgrasada en el crecimiento del conejo
}

\author{
Yanixi Acosta-Acosta ${ }^{1}$ \\ Ángel Luis La-O-Michel ${ }^{2}$ \\ Manuel Valdivié-Navarro ${ }^{3}$ \\ Anabel Maceo-Martínez ${ }^{4}$ \\ Euler Acosta-Ramos ${ }^{5}$ \\ Nelson Nixon Betancourt-Santos ${ }^{6}$ \\ Yudelkis Villalón-Moracen ${ }^{7}$ \\ Suset Hechavarría-Riviaux ${ }^{8}$
}

Fecha de recepción: 12 de marzo de 2019

Fecha de aceptación: 9 de julio de 2019

\section{Resumen}

Para evaluar la influencia de la harina de coco desgrasada (HCD) en el comportamiento productivo y el rendimiento a la canal para conejos en crecimiento, fueron elaboradas cinco dietas con niveles crecientes de esta $(0,10$, 20,30 y $40 \%$ ), las cuales fueron suministradas a 130 animales de la raza chinchilla, con un diseño completamente aleatorizado. Fueron determinados los pesos vivo inicial y final, ganancia media diaria, consumo de alimento, conversión alimenticia, rendimiento a la canal y sus cortes valiosos. El período experimental fue de 78 días. El peso vivo final de los animales durante el ciclo de ceba estuvo entre 2060 y $2242 \mathrm{~g} / \mathrm{animal}$, en todos los casos por encima de los $2000 \mathrm{~g}$, que es el establecido para el sacrificio de los animales. La ganancia media de peso osciló entre 25.4 y 28.4. La inclusión de la harina de coco desgrasada en la dieta hasta un $40 \%$ no afectó el rendimiento a la canal, sus valores promedios oscilaron entre 49.72 y $53.5 \%$. Los resultados indican que los conejos realizaron una adecuada absorción de los nutrientes al utilizar harina de coco desgrasada para su crecimiento, además se mejora el rendimiento económico del sistema de producción.

Palabras clave: animales monogástricos; cunicultura; indicadores productivos; rendimiento a la canal; subproducto del coco.

\footnotetext{
${ }^{1}$ Ph. D. Universidad de Guantánamo (Guantánamo, Cuba). yanixi@cug.co.cu.

2 Ph. D. Universidad de Guantánamo (Guantánamo, Cuba). nolo@cug.co.cu.

${ }^{3}$ Ph. D. Instituto de Ciencia Animal (Mayabeque, Cuba). mvaldivie@ica.co.cu.

4 Universidad de Guantánamo (Guantánamo, Cuba).

5 M. Sc. Universidad de Guantánamo (Guantánamo, Cuba). eulerar@cug.co.cu.

${ }^{6}$ M. Sc. Oficina Nacional de Inspección Estatal (Guantánamo, Cuba).

${ }^{7}$ M. Sc. Universidad de Guantánamo (Guantánamo, Cuba).yudelkis@cug.co.cu.

8 Ph. D. Universidad de Guantánamo (Guantánamo, Cuba). susethr@cug.co.cu.
} 


\title{
Effect of Defatted Coconut Flour on Rabbit Growth
}

\begin{abstract}
To evaluate the influence of defatted coconut flour (HCD) on the productive performance and yield to the carcass for growing rabbits, five diets were elaborated with increasing levels of this $(0,10,20,30$ and $40 \%)$, which were supplied to 130 animals of the Chinchilla breed, with a completely randomized design. The initial and final live weights, average daily gain, feed consumption, feed conversion, yield to the carcass and its valuable cuts were determined. The experimental period was 78 days. The final live weight of the animals during the fattening cycle was between 2060 and $2242 \mathrm{~g} /$ animal, in all cases above $2000 \mathrm{~g}$ which is the one established for the slaughter of the animals, the mean weight gain oscillated between 25.4 and 28.4. The inclusion of defatted coconut meal in the diet up to $40 \%$ did not affect the yield to the carcass, its average values ranged between 49.72 and $53.5 \%$. The results indicate that the rabbits made an adequate absorption of the nutrients when using defatted coconut flour for their growth, in addition the economic performance of the production system is improved.
\end{abstract}

Keywords: coconut byproduct; cuniculture; monogastrics animals; performance to the channel; productive indicators.

\section{Para citar este artículo:}

Acosta-Acosta, Y., La-O-Michel, A. L., Valdivié-Navarro, M., Maceo-Martínez, A., Acosta-Ramos, E., Betancourt-Santos, N. N., Villalón-Moracen, Y., \& HechavarríaRiviaux, S. (2019). Efecto de la harina de coco desgrasada en el crecimiento del $\begin{array}{llll}\text { conejo. Ciencia } & \text { y } & \text { Agricultura, } & \text { 16(3), }\end{array}$ https://doi.org/10.19053/01228420.v16.n3.2019.9751.

Esta obra está bajo licencia internacional Creative Commons Reconocimiento 4.0

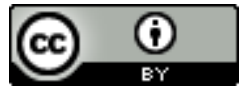




\section{INTRODUCCIÓN}

Se espera que la demanda global de carne sea un $73 \%$ mayor en 2050, y una gran parte de este aumento se producirá en los países en desarrollo (OCDE-FAO, 2014) debido a los ingresos y el rápido crecimiento de la población (Makkar et al., 2014).

Como consecuencia, la presión sobre los recursos de alimentación se ha incrementado, especialmente lo referido al crecimiento del consumo de alimento. Esto requiere realizar investigaciones utilizando recursos de alimentación alternativos, de animales asequibles y más eficientes, además de establecer sistemas de producción con el fin de satisfacer de manera sostenible la demanda creciente de carne de calidad (Khan et al., 2016).

La producción de conejos a nivel mundial se torna cada vez más importante, liderando la producción mundial Europa con $48 \%$ y Asia con un $42 \%$, en menor cuantía África y Suramérica con el 8 y $2 \%$ respectivamente, mientras que para los países de Norte y centro América las producciones no son representativas solo lo ven como una alternativa alimentaria que les permite producir carne de una calidad excelente (FAOSTAT, 2016).

La crianza de conejos (Oryctolagus cuniculus) se destaca por su rápido crecimiento, alta fertilidad, precocidad reproductiva y prolificidad, logrando desempeñar un papel importante en la alimentación humana, al poseer su carne un alto valor proteico, bajo nivel de grasa y colesterol. Por las características del sistema digestivo del conejo este puede ser alimentado con numerosos subproductos agrícolas o agroindustriales (Gidenne, 2015), al tiempo que reduce la competencia con la comida humana.

Por otra parte la carne de conejo tiene buen sabor, se digiere fácilmente, posee altas propiedades nutricionales y dietéticas, tales como alto contenido de proteínas (20-21\%), ácido linolénico, y bajos contenidos de sodio y colesterol (Dalle Zotte 2002; Dalle Zotte y Szendro 2011; Hermida et al. 2006). Las últimas propiedades hacen que la carne de conejo sea favorable para pacientes cardiovasculares y puede beneficiar la salud humana a largo plazo (Hu y Willett, 2002).

Los conejos son los convertidores de alimentación más eficientes en carne. (Taiwo et al., 2004).

El conejo es una especie que requiere concentraciones adecuadas de nutrientes, de ahí la importancia de suministrar un alimento concentrado y un suplemento que favorezca su adecuado crecimiento y reduzcan los costos por concepto de alimentación (Betancourt, 2005).

En la producción de conejos, el alimento representa aproximadamente el $60 \%$ de los costos de producción (Gidenne, Garreau, Drouilhet, Aubert \& Maertens, 2017). Sin embargo, el uso de subproductos reduce el costo de la producción del 
alimento y el impacto de los mismos en el medio ambiente (Bonaudo et al., 2014; Makkar, 2016).

Esta situación ha estimulado la búsqueda de alternativas locales, utilizando la infraestructura existente para materializar la explotación de nuevas materias primas alimenticias con la finalidad de generar patrones de producción ajustados a la realidad social y económica de cualquier entorno (Nieves, 2005).

El alto costo actual de los piensos convencionales es el reemplazo de los alimentos convencionales con alimentos no convencionales en dietas para conejos. En varios países se han utilizado subproductos agrícolas y agroindustriales incluidos en la elaboración de piensos alternativos destinados a reducir el costo del alimento y han sido evaluado por separado en varias etapas de la vida del conejo.

Aunque en el mundo se buscan varias alternativas para la formulación de dietas para la alimentación del conejo, esta se basa fundamentalmente en la utilización de materias primas importadas (maíz, soya y alfalfa) cuyos precios tienden a aumentar cada día en el mercado internacional.

Por ello es necesario identificar recursos alternativos alimenticios con el fin de sustituir parcialmente el alimento concentrado comercial en condiciones tropicales.

El cocotero (Cocos nucífera L.) es una planta arbórea distribuida en los trópicos, provee el sustento de billones de personas a través del mundo y se cultiva en más de 80 países en el trópico (Sudharmaidevi et al., 2015), siendo Indonesia y Philipinas los mayores productores (FAO, 2017). Del endospermo de su fruto se obtiene aceite como producto principal y harina como residuo (30-40\% del peso inicial).

En la industria alimenticia la harina de coco constituye uno de los subproductos más empleados en la alimentación animal por su valor nutricional (Hidalgo, 2010).

La harina de coco desgrasada es un subproducto agroindustrial procedente de la producción de aceite de coco, económico, disponible localmente, con un adecuado balance de nutrientes y se puede considerar una buena fuente de proteína (19-20 \%) y fibra bruta (14.2\%) (Acosta, Raymónd, Pérez, La O. \& Villalón, 2016). Se ha reportado su utilización en la alimentación de conejos (Vasconcelos, 2007; Aurea, 2009; Acosta et al., 2016; Acosta-Acosta, La O, Valdivié-Navarro \& CantalapiedraBello, 2018).

El objetivo de nuestro estudio fue evaluar el comportamiento productivo y los indicadores de rendimiento a la canal de conejos en ceba sometidos a variantes de alimentación donde se incorpora la harina de coco desgrasada. 


\section{MATERIALES Y MÉTODOS}

El trabajo se realizó en la instalación cunícola del Polígono Docente Investigativo del Centro de Estudio de Tecnologías Agropecuarias (CETA) perteneciente a la Facultad Agroforestal de la Universidad de Guantánamo.

Fueron utilizados 130 conejos de la raza chinchilla de 35 días de edad, con un peso promedio de $568 \mathrm{~g}$, ubicados dos animales por jaula bajo un diseño completamente aleatorizado. Los tratamientos fueron constituidos por una ración referencial y cuatro raciones por investigar, con $10 \%, 20 \%, 30 \%$ y $40 \%$ de inclusión de harina de coco desgrasada. Las variables evaluadas fueron peso vivo inicial, peso vivo final, ganancia media diaria, consumo de alimentos y conversión alimenticia.

El experimento se llevó a cabo durante 78 días; al finalizar fueron sacrificados diez animales por tratamiento y se hicieron las siguientes mediciones: peso de la canal, peso del tren anterior, peso del tren posterior, peso del lomo, peso del dorso y las costillas.

Las dietas fueron elaboradas con base en las exigencias nutricionales de los animales reportados por De Blas y Mateos (2010). Los valores de la composición porcentual de las dietas se presentan en la Tabla 1.

Tabla 1. Composición porcentual de los ingredientes en las dietas evaluadas.

\begin{tabular}{|l|c|c|c|c|c|}
\hline \multirow{2}{*}{ Ingredientes } & \multicolumn{5}{|c|}{ Inclusión de harina de coco desgrasada } \\
\cline { 2 - 6 } & $\mathbf{0} \%$ & $\mathbf{1 0} \%$ & $\mathbf{2 0} \%$ & $\mathbf{3 0} \%$ & $\mathbf{4 0} \%$ \\
\hline Harina de maíz & $\mathbf{2 4 . 7 2}$ & $\mathbf{2 3 . 9 9}$ & $\mathbf{2 3 . 0 5}$ & $\mathbf{1 6 . 6 5}$ & $\mathbf{7 . 8}$ \\
\hline Harina de alfalfa & 55.33 & 50.04 & 45.84 & 46.33 & 47.96 \\
\hline Harina de soya & 12.87 & 10.11 & 6.85 & 2.85 & 0 \\
\hline Harina de coco industrial & 0.00 & 10.00 & 20.0 & 30.0 & 40.0 \\
\hline Fosfato dicálcico & 1.06 & 0.90 & 0.73 & 0.58 & 0.45 \\
\hline Carbonato calcio & 0.00 & 0.00 & 0.00 & 0.00 & 0.17 \\
\hline Sal común & 0.30 & 0.30 & 0.30 & 0.30 & 0.30 \\
\hline Metionina & 0.12 & 0.12 & 0.12 & 0.12 & 0.12 \\
\hline Lisina & 0.00 & 0.04 & 0.11 & 0.17 & 0.20 \\
\hline Premezcla & 2.00 & 2.00 & 2.00 & 2.00 & 2.00 \\
\hline Zeolita & 1.50 & 1.00 & 1.00 & 1.00 & 1.00 \\
\hline Aceite de coco & 2.10 & 1.50 & 0 & 0 & 0 \\
\hline
\end{tabular}

\section{A. Determinaciones analíticas de los alimentos}

Los análisis químicos de las dietas se realizaron en el Instituto de Ciencia Animal. Los contenidos de materia seca, cenizas, materia orgánica, proteína bruta y fibra bruta se determinaron a partir de la metodología descrita por la Association Official Analytical Chemistry (AOAC, 1995); el fraccionamiento de la fracción fibrosa, según Van Soest, Robertson y Lewis (1991), y el calcio y el fósforo, según Herrera (1980). 


\section{B. Análisis estadístico}

El efecto de la inclusión de la harina de coco desgrasada (HCD) en la composición química de las dietas, los indicadores productivos, rendimiento a la canal y sus cortes valiosos fueron sometidos a un análisis de varianza simple; las diferencias entre las medias se determinaron por la prueba de Duncan (1955). En todos los casos se utilizó el paquete estadístico Statgraphics plus 5.1

\section{RESULTADOS Y DISCUSIÓN}

En la Tabla 2 se aprecia la similitud en cuanto al contenido nutritivo de las dietas elaboradas con la inclusión de la HCD. La MS mantuvo valores entre 87 y $89 \%$, lo cual sugiere una adecuada concentración de nutrientes, hecho que coincide con los recomendados por Retore, Silva, Toledo y Araújo (2010), que reportan valores de 86-89 \% utilizando subproductos agroindustriales en la alimentación del conejo.

El contenido de proteína bruta mostró un promedio del $18 \%$, lo cual está en correspondencia con las recomendaciones para esta especie hechas por Rodríguez (2009) y De Blas y Mateos (2010), puesto que sus principales recomendaciones fueron 14.2-18.0\%, y por Retore et al. (2010), que obtuvieron valores de $18 \%$, y por encima de Khan et al. (2016), quienes al formular dietas utilizadas en la alimentación del conejo con subproductos agroindustriales el porcentaje fue de 16 .

Las dietas con la inclusión de la HCD mostraron un excelente contenido de material celulítico con fibra bruta en el $14 \%$, coincidiendo con Trocino, García, Carabaño y Xiccato (2013), que recomiendan para dietas de conejos en la etapa de posdestete y crecimiento entre $12-14 \%$ de fibra.

Tabla 2. Composición química de dietas con niveles crecientes de harina de coco desgrasada.

\begin{tabular}{|c|c|c|c|c|c|c|}
\hline \multirow[b]{2}{*}{ Indicadores } & \multicolumn{5}{|c|}{ Inclusión de harina de coco desgrasada (HCD) } & \multirow[t]{2}{*}{ EE } \\
\hline & $0 \%$ & $10 \%$ & $20 \%$ & $30 \%$ & $40 \%$ & \\
\hline Materia seca & 88 & 89 & 87 & 89 & 87 & $0.55^{\mathrm{ns}}$ \\
\hline Proteína bruta & 18.86 & 18.68 & 18.31 & 18.35 & 18.59 & $0.21^{\text {ns }}$ \\
\hline Fibra bruta & 14.08 & 14.43 & 14.09 & 14.69 & 13.97 & $0.19^{\text {ns }}$ \\
\hline Fibra ácida detergente & $27.18^{b}$ & $27.56^{b}$ & $26.8^{b}$ & $31.7^{a}$ & $30.35^{a}$ & $0.66^{*}$ \\
\hline Fibra neutro detergente & 60.82 & 61.63 & 63.59 & 66.15 & 64.22 & $2.34^{\text {ns }}$ \\
\hline Celulosa & $23.81^{\mathrm{ab}}$ & $23.51^{\mathrm{ab}}$ & $20.40^{c}$ & $23.03^{b}$ & $25.24^{a}$ & $0.57^{*}$ \\
\hline Lignina & $2.16^{c}$ & $2.78^{c}$ & $5.51^{b}$ & $6.04^{b}$ & $7.51^{a}$ & $0.31^{*}$ \\
\hline Cenizas & $9.19^{\mathrm{b}}$ & $9.26^{\mathrm{ab}}$ & $9.39^{\mathrm{b}}$ & $9.72^{\mathrm{a}}$ & $9.86^{\mathrm{a}}$ & $0.04^{\star}$ \\
\hline Calcio & $1.13^{\mathrm{b}}$ & $1.41^{\mathrm{a}}$ & $1.20^{\mathrm{b}}$ & $0.99^{c}$ & $1.14^{\mathrm{b}}$ & $0.03^{*}$ \\
\hline Fósforo & $0.50^{\mathrm{a}}$ & $0.46^{\mathrm{ab}}$ & $0.48^{a}$ & $0.39^{\mathrm{b}}$ & $0.39^{\mathrm{b}}$ & $0.02^{*}$ \\
\hline Potasio & $1.26^{\mathrm{c}}$ & $1.26^{c}$ & $1.29^{\mathrm{b}}$ & $1.30^{\mathrm{b}}$ & $1.41^{\mathrm{a}}$ & $0.07^{*}$ \\
\hline Magnesio & $0.31^{\mathrm{c}}$ & $0.45^{a}$ & $0.28^{d}$ & $0.41^{b}$ & $0.30^{\mathrm{cd}}$ & 0.008 \\
\hline
\end{tabular}

ab Superíndice distinto en la misma fila indica diferencia significativa a $p \leq 0.05$

Los resultados sobre el comportamiento productivo de los conejos al final del período de la ceba: peso final, ganancia media diaria, consumo y conversión alimenticia se exponen en la Tabla 3. 
El peso vivo final de los animales estuvo en el rango de 2060 y $2242 \mathrm{~g} / \mathrm{animal}$, en todos los casos por encima de los $2000 \mathrm{~g}$, que es el establecido para el sacrificio de los animales; el mayor peso al sacrificio se obtuvo en los animales de la dieta control. No obstante, consideramos que la HCD constituye una materia prima atractiva, teniendo en cuenta que los animales en que se obtuvieron los pesos más bajos, que fue cuando se incluyó el $40 \%$, alcanzan el peso al sacrificio a solo 60 días de ceba, lo cual pudo estar relacionado con la calidad que presenta la proteína de la HCD.

Los resultados del peso vivo final de los animales en este estudio indican valores superiores a los obtenidos por Abubakar, Ibrahim, Yusuf, Muhammad y Adamu (2015), quienes reportaron un peso final que osciló entre 1150-1615 g con sistemas de alimentación donde se fue sustituyendo la soya como alimento proteico y utilizaron en las dietas otros subproductos agroindustriales. Al comparar los valores de peso final obtenido por los conejos en este estudio, superan a los obtenidos por Mennani et al. (2017) cuando sustituyeron parcialmente la soya y el maíz por subproductos agroindustriales como la harina de semillas de albaricoque y de rebus en Argelia, y los pesos al sacrificio oscilaron entre 1886-1996 g.

La ganancia media de peso mostró reducción y con ello diferencia significativa con respecto al control a partir de la inclusión de la HCD $20 \%$ en la dieta; no obstante, los animales de los grupos donde se incluyó el 20, 30 y $40 \%$ de HCD mostraron un alto ritmo de crecimiento con GMD superior a los $25 \mathrm{~g}$, básicamente promovido por el adecuado consumo de nutrientes que realizaron los animales. Esto confirma que las características específicas de las materias primas utilizadas en la elaboración de los piensos poseen un papel determinante en el consumo y el desempeño de los animales (Lebas, 2004).

Makinde (2016) evaluó el efecto de la utilización de diferentes subproductos agroindustriales en el crecimiento del conejo, con valores en la composición de nutrientes de las dietas muy similares a las nuestras; los valores de ganancia de peso (9.86-19.35 g) se comportaron inferiores a los obtenidos en este estudio (25.4- $28.4 \mathrm{~g})$.

El consumo de alimento mostrado por los animales revela que este disminuye a partir de la incorporación del $30 \%$ de HCD, mostrando diferencia significativa de los tratamientos con 30 y $40 \%$ de inclusión de HCD con respecto a los demás. Sin embargo, la cantidad de alimento consumido coincide con lo reportado por Vasconcelos (2007), quien indicó que disminuye el consumo de alimentos en conejos cuando se incluye en un $25 \%$ en sus dietas.

La conversión alimenticia mostró valores desde 3.64 hasta $3.90 \mathrm{~g}$ de alimento/g, con diferencias significativas entre la ración referencial y las dietas con niveles superiores al $20 \%$ de inclusión de HCD. No obstante, se obtuvieron excelentes índices de conversión alimenticia, teniendo en cuenta que sus valores fueron inferiores a los $4 \mathrm{~g}$ de alimento/g de incremento de carne. Los promedios de 
conversión alimenticia reportados por Vasconcelos (2007) fueron de 3.29 inferiores a los obtenidos en este trabajo, con efecto positivo para este indicador con la inclusión de hasta el $25 \%$ de la harina de coco en la dieta.

Los resultados obtenidos en los diferentes indicadores productivos del conejo en crecimiento en este estudio están en correspondencia con los informes de literatura sobre los niveles de inclusión de estos y otros subproductos agrícolas en dietas de conejos en diferentes países, sin ocasionar efectos adversos sobre el rendimiento (Orunmuyi et al., 2006; Adeyemi et al., 2014; Ansah, Francis, Seth \& Rockson, 2014; Makinde, Sikiru, Ajibade \& Johnson, 2017; Mennani et al., 2017).

Tabla 3. Comportamiento de los indicadores productivos de conejos alimentados con niveles crecientes de harina de coco desgrasada en la dieta.

\begin{tabular}{|c|c|c|c|c|c|c|}
\hline \multirow[b]{2}{*}{ Indicadores } & \multicolumn{5}{|c|}{ Inclusión de harina de coco desgrasada } & \multirow[b]{2}{*}{ EE } \\
\hline & $0 \%$ & $10 \%$ & $20 \%$ & $30 \%$ & $40 \%$ & \\
\hline Peso vivo inicial, $g$ & 569 & 567 & 571 & 566 & 569 & $3.07^{\mathrm{ns}}$ \\
\hline Peso vivo final, $\mathrm{g}$ & $2242^{a}$ & $2178^{b}$ & $2164^{\mathrm{b}}$ & $2125^{\mathrm{c}}$ & $2060^{d}$ & $9.31^{*}$ \\
\hline Ganancia media diaria, $\mathrm{g}$ & 28. $4 a$ & $27.3^{\mathrm{ab}}$ & $27,0^{\mathrm{bc}}$ & $26,3^{c}$ & $25,4^{c}$ & $0,15^{\star}$ \\
\hline Consumo de alimento, $\mathrm{g}$ & $102.6^{a}$ & $102.6^{a}$ & $103.5^{\mathrm{a}}$ & $101,1^{\mathrm{b}}$ & $99,2^{\mathrm{c}}$ & $0.49^{*}$ \\
\hline Conversión alimenticia & $3,63^{c}$ & $3,76^{b}$ & $3,83^{a b}$ & $3,83^{a b}$ & $3.90^{\mathrm{a}}$ & $0,03^{*}$ \\
\hline
\end{tabular}

ab Superíndice distinto en la misma fila indica diferencias significativas a $p \leq 0.05$

Los valores obtenidos en el rendimiento a la canal (Figura 1) no mostraron diferencias significativas a $(p \leq 0.05)$ entre los tratamientos, sus valores promedios oscilaron entre 49.72 y $53.5 \%$ y están en correspondencia con los pesos promedio de los animales al sacrificio y a la velocidad de crecimiento, ya que si la velocidad de crecimiento es rápida, el rendimiento aumenta, debido a la reducción de la proporción en el peso vivo de tejidos precoces y entre ellos el aparato digestivo (Ouhayoun, 1991). Los valores promedios del rendimiento de las carcasas obtenidas son similares a los informados por Ansah et al. (2014), los cuales reflejaron valores entre $49-55 \%$, y por debajo de los conseguidos por Mennani et al. (2017) y Theodore et al. (2017), quienes indican el $61 \%$ utilizando diversos sistemas de alimentación en conejos, donde incluyen subproductos agroindustriales.

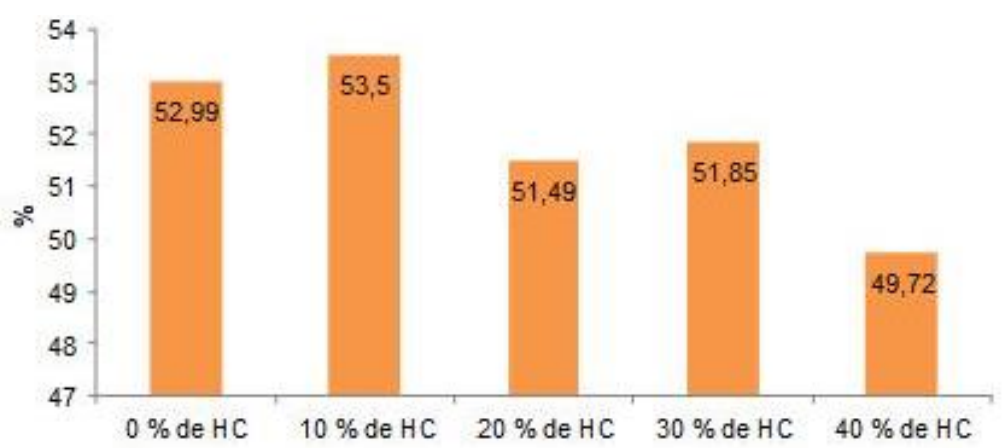

Fig. 1. Rendimiento a la canal de conejos alimentados con niveles crecientes de harina de coco desgrasada. 
La Tabla 4 muestra el rendimiento de los cortes valiosos de la canal. Se observa que no hubo diferencias significativas $(p \leq 0.05)$ para las porciones tren posterior, dorso-lomo y las costillas; mientras que la región del tren anterior mostró diferencias significativas entre los grupos que consumieron el 40 y el $20 \%$ de HCD. Los resultados evidencian, además, que las regiones de mejor distribución de carne se encuentran en el tren posterior y el dorso-lomo; resultados similares a los de La O (2007), quien encontró que los animales presentaron una distribución uniforme con respecto a la canal y precisamente en el lomo es donde se marcan las diferencias con los mayores valores porcentuales para los sistemas Teramnus labialis-caña-girasol, Ipomoea batata caña-girasol y Phyla nodiflora-caña-girasol; los valores más bajos fueron para los animales del sistema Hibiscus rosa-sinensiscaña-girasol.

Tabla 4. Comportamiento de los indicadores de calidad de la canal de conejos alimentados con niveles crecientes de harina de coco desgrasada.

\begin{tabular}{|l|l|l|l|l|l|l|}
\hline \multirow{2}{*}{ Indicadores } & \multicolumn{5}{|c|}{ Inclusión de harina de coco desgrasada } & \multirow{2}{*}{ EE } \\
\cline { 2 - 7 } & $\mathbf{0} \%$ & $\mathbf{1 0} \%$ & $\mathbf{2 0} \%$ & $\mathbf{3 0} \%$ & $\mathbf{4 0} \%$ & \\
\hline Tren anterior & $17.71^{\mathrm{ab}}$ & $18.26^{\mathrm{ab}}$ & $17.07^{\mathrm{b}}$ & $17.98^{\mathrm{ab}}$ & $18.74^{\mathrm{a}}$ & $0.47^{*}$ \\
\hline Tren posterior & 35.17 & 34.32 & 35.52 & 33.87 & 34.53 & $1.04^{\mathrm{ns}}$ \\
\hline Dorso-lomo & 27.64 & 26.76 & 26.08 & 28.2 & 27.06 & $1.12^{\mathrm{ns}}$ \\
\hline Costillas & 20.11 & 20.67 & 20.41 & 19.25 & 19.99 & $0.62^{\mathrm{ns}}$ \\
\hline
\end{tabular}

ab Superíndice distinto en la misma fila indica diferencias significativas a $p \leq 0.05$

\section{CONCLUSIÓN}

La inclusión de la harina de coco desgrasada como ingrediente en la dieta de los conejos en ceba en niveles de 10,20,30 y $40 \%$ promovió altos consumos de MS y permitió satisfacer las necesidades de PB, ED, FB y minerales, no afecto el consumo de nutrientes.

Las variantes de alimentación evaluadas permitieron lograr peso al sacrificio a los 78 días de ceba entre 2060 y 2242g, ganancia media de peso de 25,4 hasta 28,4 y conversión alimenticia entre 3,69 y 3,90.

Los valores promedios del rendimiento a la canal de los animales alimentados con dieta donde se incluyó la harina de coco desgrasada de 10 hasta el $40 \%$ oscilaron entre 49,72 y $53,5 \%$.

\section{REFERENCIAS}

Abubakar, M., Ibrahim, U., Yusuf, A. U., Muhammad, A. S., \& Adamu, N. (2015). Growth Performance, Carcass and Organ Characteristics of Growing Rabbits Fed Graded Levels of Moringa Oleifera Leaf Meal in Diets. Journal of Pure and Applied Sciences, 8(2), 7-9. https://doi.org/10.4314/bajopas.v8i2.2.

Acosta, Y., Raymónd, L. M., Pérez, G. N., La O, M. A., \& Villalón, M. Y. (2016). Inclusión de diferentes niveles de harina de coco industrial en dietas para conejos. Hombre, Ciencia y Tecnología, 20(2), 99106.

Acosta-Acosta, Y., La O, M. A. L., Valdivié-Navarro, M. I., \& Cantalapiedra-Bello, J. (2018). Digestibilidad de dietas con niveles crecientes de harina de coco desgrasada en conejos en crecimiento. Ciencia $y$ Agricultura, 15(1), 45-51. https://doi.org/10.19053/01228420.v15.n1.2018.7755. 
Efecto de la harina de coco desgrasada en el crecimiento del conejo

Adeyemi, O. A., Adesanya, P. A., Eniolorunda, O. O., Sogunle, O. M., Egbeyale, L. T., \& Njoku, C. P. (2014). Performance of Growing Rabbits Fed Diets Containing Fermented and Unfermented Cassava Leaf: Peel Meal Mix as Replacement for Maize. Malaysian Journal of Animal Science, 17(1), 61-72.

Ansah, T., Francis, K., Seth, J. N., \& Rockson, T. K. (2014). Growth, Carcass and Blood Profile of Rabbits Fed Agro-Industrial By-Products in Northern Ghana. Journal of Science, Technology and Development, $1(1)$.

Association Official Analytical Chemistry (1995). Official Methods of Analysis. (11 ed.). Washington DC.: AOAC.

Betancourt, L. (2005). Evaluación del contenido de ácidos grasos en la canal de conejos alimentados con morera Morus alba. Tesis Doctorado, Universidad de la Salle, Bogotá, D.C., Colombia.

Bonaudo, T., Bendahan, A. B., Sabatier, R., Ryschawy, J., Bellon, S., Leger, F., Magda, D., \& Tichit, M. (2014). Agroecological Principles for the Redesign of Integrated Crop-Livestock Systems. Europ. J. Agronomy, 57, 43-51. https://doi.org/10.1016/j. eja.2013.09.010.

Dalle Zotte, A., \& Szendro, Z. (2011). The role of rabbit meat as functional food. Meat Science, 88, 319-331. https://doi.org/10.1016/j.meatsci.2011.02.017.

Dalle Zotte, A. (2002). Perception of rabbit meat quality and major factors influencing the rabbit carcass and meat quality. Livestock Production Science, 75(1), 11-32. https://doi.org/10.1016/S03016226(01)00308-6.

De Blas, C., \& Mateos, G.G. (2010). Feed formulation. In C. De Blas \& J. Wiseman (eds.) Nutrition of the Rabbit (pp. 222-232). Cambridge: CAB International.

Duncan D. B. (1955). Multiple Range Test. Biometrics, 11, 1-41.

FAO. (2017). Estatistics Divition. Disponible en: https://www.fao.org/index es.htm/2017.

FAOSTAT (2016). Agriculture Data. FAO, Rome. Disponible en: https://faostat.fao.org.

Hidalgo, K. (2010). El mundo de las Plantas. Disponible en: https://www.botanical-online.com.

Gidenne T., García J., Lebas F., \& Licois, D. (2010). Nutrition and Feeding Strategy: Interactions with Pathology. In: C. de Blas, J. Wiseman (Eds.), Nutrition of the Rabbit, 2nd Edition. CAB International. Oxford, UK.

Gidenne, T., Garreau, H., Drouilhet, L., Aubert, C., \& Maertens, L. (2017). Improving Feed Efficiency in Rabbit Production. A Review on Nutritional, Technico-Economical, Genetic and Environmental Aspects. Animal Feed Sci. Technol., 225, 109-122. https://doi.org/10.1016/i.anifeedsci.2017.01.016.

Gidenne, T. (2015). Le lapin. De la biologie à l'élevage. Quae Ed, Versailles, France, p. 270.

Hermida, M., Gonzalez, M., Miranda, M., \& Rodriguez-Otero, J. L. (2006). Mineral analysis in rabbit meat from Galicia (NW Spain). Meat Science, 73, 635-639.

Herrera, R. S. (1980). Análisis químico del pasto. Metodología para las tablas de su composición. La Habana, Cuba: EDICA, Instituto de Ciencia Animal, San José de las Lajas.

Hu, F. B., \& Willett, W. C. (2002). Optimal diets for prevention ofcoronary heart disease. Journal of the American Medical Association, 288, 2569-2578.

Khan, K., Khan, S., Khan, R., Sultan, A., Ahmad, N. K., \& Ahmad, N. (2016). Growth Performance and Meat Quality of Rabbits under Different Feeding Regimes. Trop Anim Health Prod., 48, 1661-1666. https://doi.org/10.1007/s11250-016-1140-4.

La O A.L. (2007). Alimentación de conejos (Oryctolagus cunículus) con follajes, caña, girasol. (Tesis en opción al grado científico de doctor en ciencias veterinarias). ICA, La Habana, Cuba.

Ciencia y Agricultura (Cien. Agri.) Vol. 16 (3). L-ISSN 0122-8420. elSSN 2539-0899.

Septiembre-Diciembre 2019, pp. 47-59. Tunja (Boyacá) - Colombia. DOI:

https://doi.org/10.19053/01228420.v16.n3.2019.9751 
Yanixi Acosta-Acosta, Ángel Luis La-O-Michel, Manuel Valdivié-Navarro, Anabel Maceo-Martínez, Euler Acosta-Ramos, Nelson Nixon Betancourt-Santos, Yudelkis Villalón-Moracen, Suset Hechavarría-Riviaux

Lebas, F. (2004). Reflections on Rabbit Nutrition with a Special Emphasis on Feed Ingredients Utilization. In 8th World Rabbit Congress. México.

Makinde, O. J. (2016). Growth Performance, Carcass Yield and Blood Profiles of Growing Rabbits Fed Concentrate Diet Supplemented with White Lead Tree (Leucaena Leucocephala) or Siratro (Macroptilium atropurpureum) Leaves in North Central Nigeria. Trakia Journal of Sciences, (1), 80-86.

Makinde, O., Sikiru, J., Ajibade, A., \& Johnson, A. (2017). Effects of Different Agro Industrial by-Products on the Growth Performance, Carcass Characteristics and Blood Profiles of Growing Rabbits. International Journal of Research in Agriculture and Forestry, 4(7), 1-8.

Makkar, H. P., Tran, G., Heuzeand, V. Y., \& Ankers, P. (2014). State-of-theart on use of insects as animal feed. Animal Feed Science and Technology, 197, 1-33.

Makkar, H. P. S. (2016). Smart Livestock Feeding Strategies for Harvesting Triple Gain - the Desired Outcomes in Planet, People and Profit Dimensions: A Developing Country Perspective. Anim. Prod. Sci., 56, 519-534. https://doi.org/10.1071/AN15557.

Mennani, A., Arbouche, R., Arbouche, Y., Montaigne, E., Arbouche, F., \& Arbouche, S.H. (2017). Effects of the Incorporation of Agroindustrial By-products in the Diet of New Zealand Rabbits: Case of Date Rebus and Apricot Flour. Veterinary Vet World. 10(12), 1456-1463. https://doi.org/10.14202/vetworld.2017.1456-1463.

Nieves, D. (2005). Forrajes promisorios para la alimentación del conejo en Venezuela. Valor nutricional. Alimentación no convencional para monogástricos en el trópico. En: VIII Encuentro de Nutrición de animales monogástricos, Guanare. Venezuela.

OECD-FAO (2014). Agricultural Outlook: 2014-2023. Disponible en: http://www.oecd.org/site/oecdfaoagriculturaloutlook.

Orunmuyi, M., Bawa, G. S., Adeyinka, F. D., Daudu, O. M., \& Adeyinka, I. A. (2006). Effects of Graded Levels of Palm-Kernel Cake on Performance of Grower Rabbits. Pakistan Journal of Nutrition, 5(1), 71-74. https://doi.org/10.3923/pjn.2006.71.74.

Ouhayoun, J. (1991). La calidad de la carne de conejo. Boletín de Cunicultura, 14(55), 31-36.

Retore, M., Silva, L. P. Toledo, G. S. P., \& Araújo, I. G. (2010). Efeito da fibra de coprodutos agroindustriais e sua avaliação nutricional para coelhos. Arq. Bras. Med. Vet. Zootec., 62(5), 1232-1240. https://doi.org/10.1590/s0102-09352010000500028.

Rodríguez, J. M. (2009). Influencia del nivel de fibra y proteína en la ración sobre la mortalidad, crecimiento e índices de conversión de conejos en cebo. En VII Simposio de Cunicultura. Asociación Española de Cunicultura (ASESCU). Santiago de Compostela, España.

Sudharmaidevi C. R., Vinith, V., \& Kavitha, G. V. (2015). Effect of potassium-sodium interaction on foliar nutrient concentration and nut quality of coconut (Cocos nucifera). Malaysian Journal of Soil Science, $19,107-114$.

Taiwo, V. O., Afolabi, O. O., \& Adegbuji, A. O. (2004). Effect of Thevetieperuviana seed cake based diet on the growth, haematology and tissues of rabbit. Tropical and Subtropical Agro-ecosystem, 4, 7-14.

Van Soest, P. J., Robertson, J. B., \& Lewis, B. A. (1991). Methods for Dietary Fibre, Neutral Detergent Fiber, and Non-Starch Polysaccharides in Relation to Animal Nutrition. J. Dairy Sci., 74, 3583-3597. https://doi.org/10.3168/jds.S0022-0302(91)78551-2.

Vasconcelos, C. A. (2007). Utilização do Farelo de Côco em dietas para coelhos destinados ao abate. (Dissertação de Mestrado). Fortaleza-Ceará: Universidade Federal do Ceará Centro de Ciências Agrárias. Programa de Pós-graduação em Zootecnia.

Ciencia y Agricultura (Cien. Agri.) Vol. 16 (3). L-ISSN 0122-8420. elSSN 2539-0899.

Septiembre-Diciembre 2019, pp. 47-59. Tunja (Boyacá) - Colombia. DOI: https://doi.org/10.19053/01228420.v16.n3.2019.9751 
Efecto de la harina de coco desgrasada en el crecimiento del conejo

Theodore, S., Vitohekpon, T., Traore, I., Alkoirte, Is-Haquou D., \& Guy, A. (2017). Evaluation of the carcass yield of rabbits in farms in Southern Benin carcass yield of rabbit. Agricu.J.,12(3-6), 11-14.

Trocino, A., García, J., Carabaño, R., \& Xiccato, G. (2013). A Meta-Analysis on the Role of Soluble Fibre in Diets for Growing Rabbits. World Rabbit Sci., 21, 1-15. https://doi.org/10.4995/wrs.2013.1285.

Ciencia y Agricultura (Cien. Agri.) Vol. 16 (3). L-ISSN 0122-8420. elSSN 2539-0899.

Septiembre-Diciembre 2019, pp. 47-59. Tunja (Boyacá) - Colombia. DOI: 